\title{
Quantification of friction force reduction induced by obstetric gels
}

\section{Journal Article}

Author(s):

Riener, Robert; Leypold, Kerstin; Brunschweiler, Andreas; Schaub, Andreas; Bleul, Ulrich; Wolf, Peter (D)

Publication date:

2009

Permanent link:

https://doi.org/10.3929/ethz-b-000017932

Rights / license:

In Copyright - Non-Commercial Use Permitted

Originally published in:

Medical \& Biological Engineering \& Computing 47(6), https://doi.org/10.1007/s11517-009-0460-1 


\title{
Quantification of friction force reduction induced by obstetric gels
}

\author{
Robert Riener · Kerstin Leypold • \\ Andreas Brunschweiler · Andreas Schaub • \\ Ulrich Bleul · Peter Wolf
}

Received: 13 October 2008/ Accepted: 2 February 2009/Published online: 24 February 2009

(C) International Federation for Medical and Biological Engineering 2009

\begin{abstract}
The objective of this study was to quantify the reduction of friction forces by obstetric gels aimed to facilitate human childbirth. Lubricants, two obstetric gels with different viscosities and distilled water, were applied to a porcine model under mechanical conditions comparable to human childbirth. In tests with higher movement speeds of the skin relative to the birth canal, both obstetric gels significantly reduced dynamic friction forces by 30 $40 \%$ in comparison to distilled water. At the lowest movement speed, only the more viscous gel reduced dynamic friction force significantly. In tests modifying the dwell time before a movement was initiated, static friction forces of trials with highly viscous gel were generally lower than those with distilled water. The performed biomechanical tests support the recommendation of using obstetric gels during human childbirth. Using the presented test apparatus may reduce the amount of clinical testing required to optimize gel formulation.
\end{abstract}

Keywords Lubrication - Mechanical testing · Movement speed · Dwell time

R. Riener $(\bowtie) \cdot$ K. Leypold · A. Brunschweiler $\cdot$ P. Wolf Department of Mechanical and Process Engineering, ETH Zurich, Tannenstrasse 1, E4,

8092 Zurich, Switzerland

e-mail: riener@mavt.ethz.ch

P. Wolf

e-mail: wolf@mavt.ethz.ch

A. Schaub

Happy Child Birth Holding AG, Basel, Switzerland

U. Bleul

Clinic of Reproductive Medicine,

Vetsuisse-Faculty University Zurich, Zurich, Switzerland

\section{Introduction}

A prolonged second stage of labor has been shown to be a risk factor for pelvic floor injury [5, 6]. A prolonged second stage of labor was further associated with increased maternal morbidity and operative delivery rates in nulliparous women [4], and with increased maternal and neonatal morbidity in multiparous women [5]. Various procedures were investigated with respect to a shortening of the second stage of labor and the protection of perineal integrity; however, results were inconsistent: Some studies showed the benefit of perineal massage to protect perineal trauma [7], whereas other studies showed no influence on perineal integrity $[2,13]$. Studies also confirmed the benefit of a lateral position for birth to reduce perineal trauma [3], whereas other studies confirmed that different positions during childbirth had neither an effect on the duration nor on the perineal integrity [7, 14-16].

The use of a lubricant to facilitate vaginal childbirth is not yet a standard procedure in human obstetrics [8], although it is a golden standard procedure in veterinary obstetrics [1]. Just recently, obstetric gels for labor facilitation in humans have been developed and brought onto the market. In a first randomized controlled study, these obstetric gels significantly shortened the duration of the second stage of labor and significantly reduced the risk for perineal tears in nulliparous women [12].

While clinical trials are essential to show the in vivo benefit of obstetric gels, appropriate mechanical technologies may demonstrate the underlying mechanisms of labor facilitation by quantifying the friction properties of obstetric gels. Our group has therefore designed an apparatus that determines friction properties of lubricants applied to a porcine model (birth canal and skin) under mechanical conditions comparable with human childbirth. 
In this study, this apparatus was used to quantify the reduction of friction forces when the porcine model was lubricated with an obstetric gel as compared to distilled water. Distilled water was used as the reference lubricant, since amniotic liquor mainly consists of pure water. The samples could easily be cleaned with water, and the inert characteristic of water reduces the bias of using it as a lubricant before using an obstetric gel. Two different obstetric gels were applied, and the conditions of the experiments were modified with respect to two important clinical parameters: (1) the movement speed of the skin relative to the birth canal, and (2) the time the skin stays in a resting position on the birth canal before the movement is initiated, the so-called dwell time. Since expected friction forces are not deducible from simple laws like that of Coulomb, modifying the movement speed is reasonable. Furthermore, during pilot studies, it became evident that a theoretical function of friction force relative to movement will not be seen in our experiments; thus, we decided to vary dwell time as well.

The presented technology is not only suited to measure the reduction of friction forces by obstetric gels but also to differentiate friction forces depending on specific gel formulations. The technology may therefore have the potential (1) to reduce the number of clinical trials, which are needed to optimize obstetric gel formulations, (2) to emphasize which obstetric gel should be used for the different stages of human childbirth, and (3) to contribute to the patients' acceptance of obstetric gels use during human childbirth facilitation.

\section{Materials and methods}

\subsection{Lubricants}

The samples were lubricated with distilled water (as a reference) and two obstetric gels (gel A: Dianatal ${ }^{\circledR}$ stage I; gel B: Dianatal ${ }^{\circledR}$ stage II; both gels: Happy Child Birth AG, Basel, Switzerland). These gels have the same ingredients (distilled water, propylene glycol and carbomers), but in different quantities, i.e., gel A is composed of $76 \%$ distilled water and is of high viscosity (dynamic viscosity of $15 \mathrm{~Pa} \mathrm{~s}$ ), while gel $\mathrm{B}$ is composed of $81 \%$ distilled water and is of low viscosity (dynamic viscosity of $5 \mathrm{~Pa} \mathrm{~s}$ ). The ingredients of both gels also include thickening agents, but no preservatives.

\subsection{The measurement apparatus}

The designed apparatus consists of a linear drive, actuated by a high-precision motor (Pollux drive type 2, iTK Dr. Kassen, Lahnau, Germany) to generate a horizontal movement, a force-sensing unit, a parallel bar mechanism, a pan to place vertical loads and a sample holder to fix the tissue probe (Fig. 1). The force-sensing unit is comprised of a high-precision horizontal force transducer (W-AL $3 \mathrm{~kg}$, Lorenz Messtechnik, Alfdorf, Germany) and a frictionless linear bearing (BWU40100, IKO Nippon Thompson, Düsseldorf, Germany), which is required to decouple the horizontal force from the vertical load in the transducer. The parallel bar guarantees a parallel contact between the stamp and sample holder, independent of the sample thickness. The device can bear a maximum normal force of $500 \mathrm{~N}$ and measure a maximum friction force of $30 \mathrm{~N}$ with a maximum error of $0.1 \mathrm{~N}$. The movement velocity is adjustable in the range of $1-420 \mathrm{~cm} / \mathrm{h}$. Contact pressures can be modified in the range of $20 \mathrm{~Pa}-1.25 \mathrm{MPa}$. Contact areas can be in the range of $4-100 \mathrm{~cm}^{2}$. The maximum movement distance of the sample holder is about $20 \mathrm{~cm}$. The construction of the measurement device allows many different types of organic and synthetic tissue models to be mounted and tested.

\subsection{The mammal model}

The mammal model consisted of fresh vaginal and dermal tissues of pigs directly obtained from a local abattoir. The clean-shaven dermal tissue was fixed to the stamp by bar clamps. The vaginal tissue was dissected from surrounding

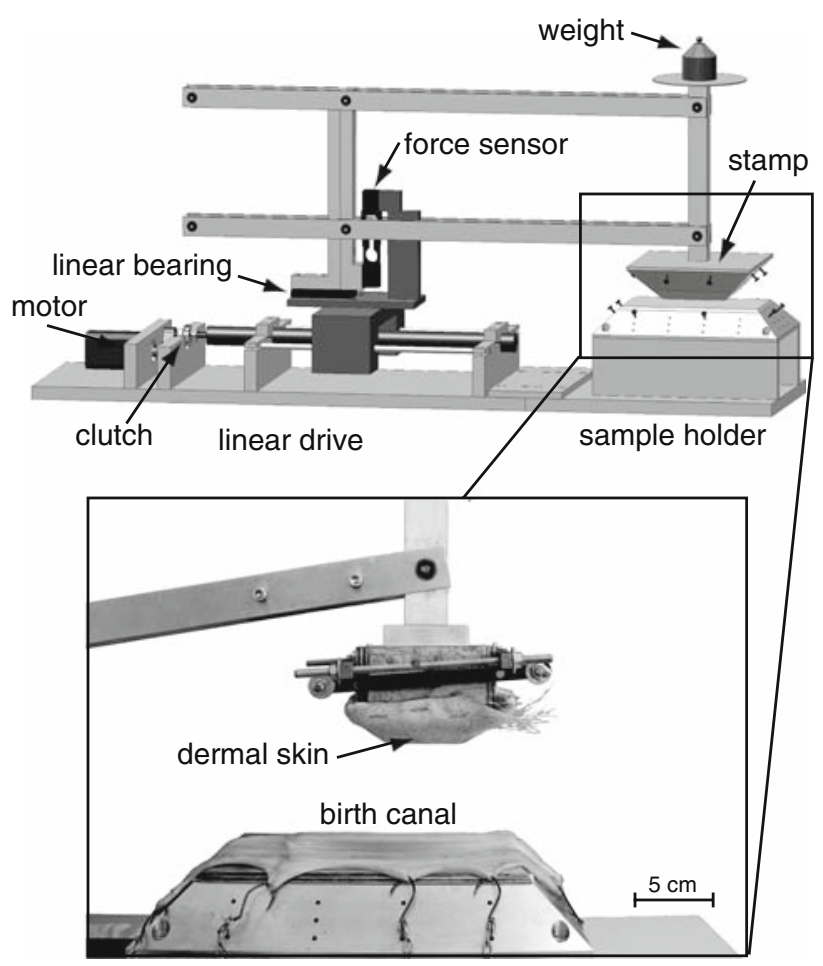

Fig. 1 Sketch of the measurement device with a photograph showing the sample holder in detail 
tissues. Then, it was stretched over the lower device in an orientation facilitating a physiological movement direction of the stamp. Finally, the vaginal tissue was fixed by fishhooks so that there were no wrinkles across the surface.

During all measurements, the room temperature was between $22^{\circ}$ and $24^{\circ} \mathrm{C}$, and relative humidity was between 25 and $35 \%$.

\subsection{Definition of test conditions}

The movement distance of the clean-shaven dermal tissue relative to the birth canal was set to $5 \mathrm{~cm}$. To reduce the effects of sample inhomogeneity, i.e., variations in thickness and surface properties, the maximum movable size of dermal tissue was used (approximately $25 \mathrm{~cm}^{2}$ ). Further test conditions were defined in accordance with the results of pilot studies investigating Gel A with the presented setup. Details of these pilot studies are given elsewhere [10] and a comprehensive summary follows.

The first pilot studies showed that lubrication on the same sample after the use of obstetric gel affected the friction force, since the obstetric gel cannot be completely removed from the vagina without damaging the tissue. In contrast, the reference lubricant (distilled water) can be removed from the birth canal without any consequences on subsequent measurements. Thus, samples were lubricated and tested with $11 \mathrm{ml}$ of water first, cleaned, and then lubricated and tested with $4 \mathrm{ml}$ of obstetric gel. The lubrication volume of gel was based on the recommendation for clinical use. The lubrication volume of water was greater due to its limited bond to the sample. Both lubrications were evenly distributed over the sample.

Further pilot studies were performed with contact pressures between 10 and $45 \mathrm{kPa}$ (steps of $5 \mathrm{kPa}$ ). Within this range of pressures, a linear dependence of the friction force on the contact pressure was found, but with different slopes for distilled water and for obstetric gel. During trials with the distilled water, an increase of $10 \mathrm{kPa}$ contact pressure resulted in an increase of approximately $1.5 \mathrm{~N}$ friction force, whereas during trials with obstetric gel, an additional $10 \mathrm{kPa}$ resulted only in an increase of approximately $0.5 \mathrm{~N}$. Thus, friction forces of studies with a constant contact pressure can be extrapolated to other contact pressures of interest. In the present work, the contact pressure was set to $30 \mathrm{kPa}$ simulating the pressure usually found between the human fetal head and cervix [11]. It was assumed that under this pressure, friction forces will not depend on the deformation of both the fetus' body and birth canal.

An obvious relationship between friction force and movement velocity (varied between 10 and $400 \mathrm{~cm} / \mathrm{h}$ ) was not found during the pilot studies. As a consequence, in the first part of the presented study, lubricants (distilled water and both obstetric gels) were tested on 36 samples at three different movement velocities $(10,50,100 \mathrm{~cm} / \mathrm{h})$ covering short-term maximal velocities during human childbirth. In the second part of the study, the movement velocity was kept constant at $50 \mathrm{~cm} / \mathrm{h}$, and 20 samples were tested after defined dwell times ranging from 30 to $600 \mathrm{~s}$, see Table 1.

\subsection{Data acquisition and analysis}

The friction force was recorded with a sampling rate of $10 \mathrm{~Hz}$.

Each sample was tested with distilled water first: After one initial test trial, three test trials were performed. After these trials with distilled water, the sample was cleaned, then lubricated with one of the obstetric gels and again tested three times. Samples investigated after a dwell time of $300 \mathrm{~s}$ were tested only two times with each lubricant in order to reduce the effects of dehydration. In the same context, samples were tested only once with distilled water and one of the gels after a dwell time of $600 \mathrm{~s}$.

Three regions can be distinguished within the friction force plots (Fig. 2). After initiation of movement, static friction dominates, where the force increases with a rather high slope (Fig. 2, left region). After reaching a certain threshold, static friction changes into dynamic friction, which is characterized by a smaller slope (Fig. 2, middle region). Occasionally at the end of the movement, the slope of the friction force increased again due to a swelling bulge of tissue that arose in front of the moving stamp (Fig. 2, right region).

The maximum friction force measured during the first $0.1 \mathrm{~cm}$ of movement was defined as a quantity of static friction (Fig. 3). This quantity was calculated for the trials of the test series with different dwell times. The dynamic

Table 1 Summary of the test conditions that were modified

\begin{tabular}{lccc}
\hline Sample id & $\begin{array}{l}\text { Velocity } \\
(\mathrm{cm} / \mathrm{h})\end{array}$ & $\begin{array}{l}\text { Dwell time } \\
(\mathrm{s})\end{array}$ & Lubricant \\
\hline $1-6$ & 10 & 0 & Gel A \\
$7-12$ & 10 & 0 & Gel B \\
$13-18$ & 50 & 0 & Gel A \\
$19-24$ & 50 & 0 & Gel B \\
$25-30$ & 100 & 0 & Gel A \\
$31-36$ & 100 & 0 & Gel B \\
$37-41$ & 50 & 30 & Gel A \\
$42-46$ & 50 & 100 & Gel A \\
$47-51$ & 50 & 300 & Gel A \\
$52-56$ & 50 & 600 & Gel A \\
\hline
\end{tabular}

During the test series with modified movement velocities, six samples were used for each condition. During the test series with modified dwell time, five samples were available for each condition 


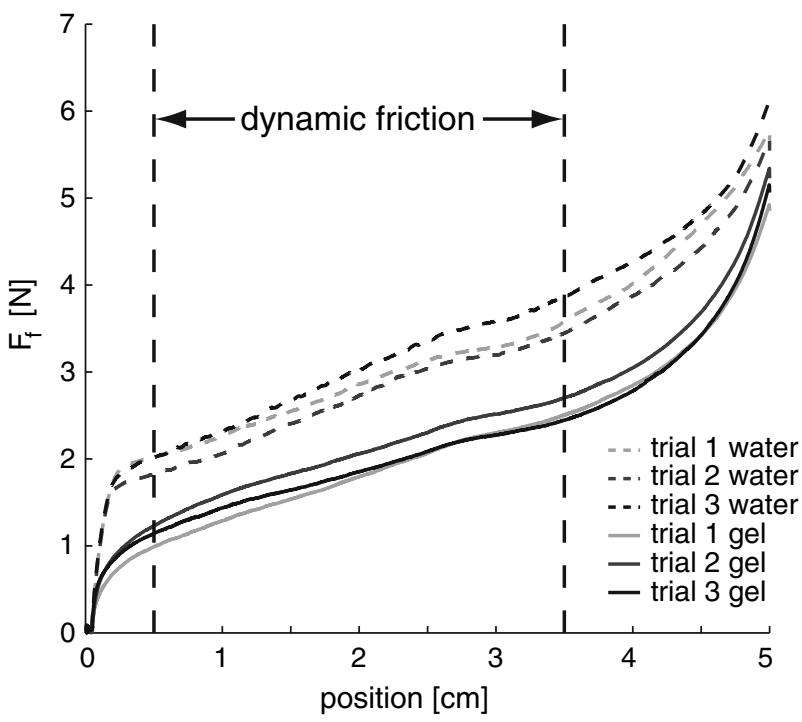

Fig. 2 Measured friction forces $F_{\mathrm{f}}$ on one sample of the test series with different movement velocity. The recorded friction forces were averaged within the vertical dashed lines to determine the dynamic friction force of the current trial

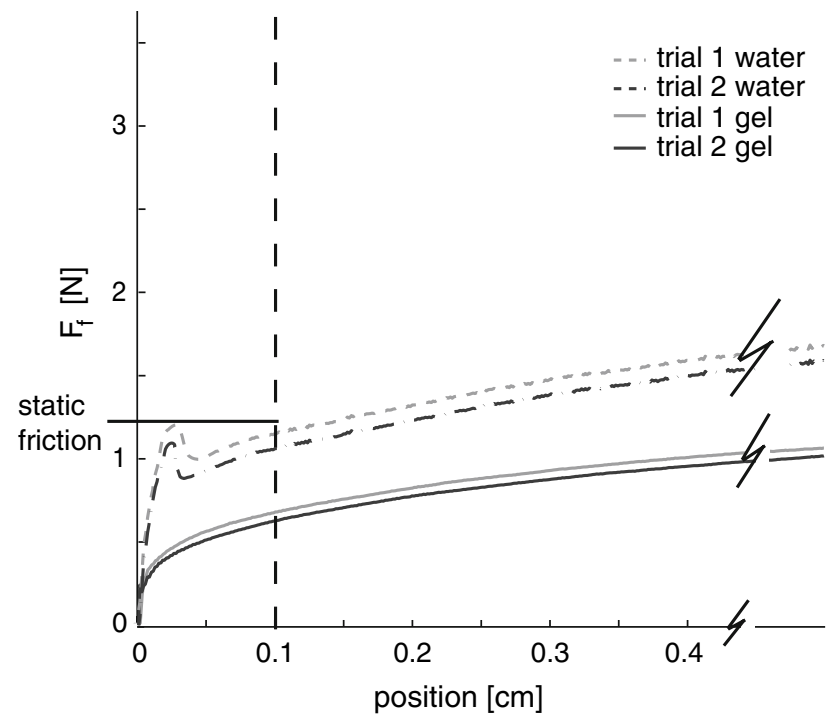

Fig. 3 Measured friction force $F_{\mathrm{f}}$ on one sample of the dwell time tests. The maximum friction force during the first millimeter was used to determine the static friction force of the current trial; this is exemplified on the first trial with water

friction was defined as the average friction force over a movement distance of $3 \mathrm{~cm}$ (middle region) starting after the initial slope, i.e., after the static friction effects disappeared (Fig 2). This quantity was calculated for the trials of the test series with different movement velocities.

For each sample, the friction forces of the repeated trials with distilled water and obstetric gel were separately averaged. This resulted in a pair of friction forces for each

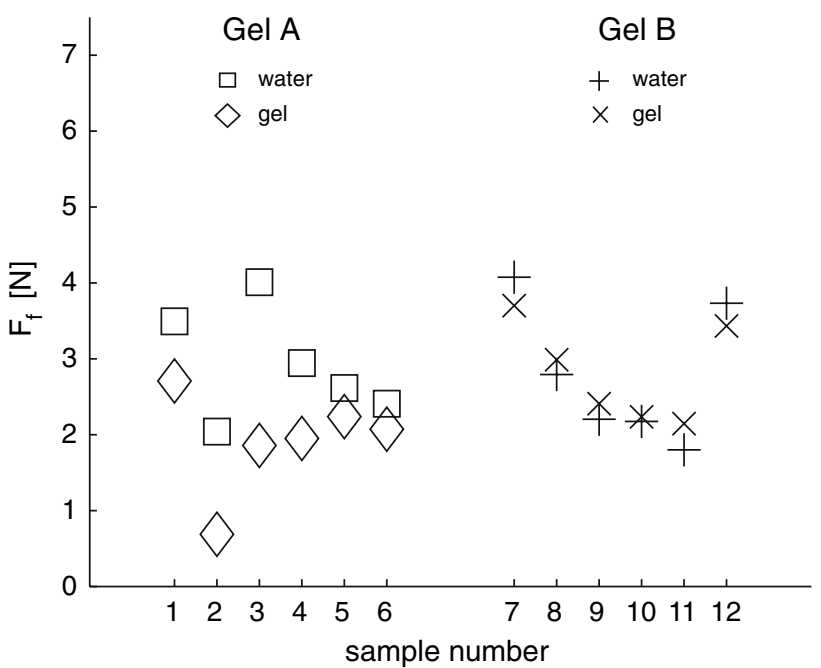

Fig. 4 Measured friction forces $F_{\mathrm{f}}$ for each sample moved at $10 \mathrm{~cm} /$ $\mathrm{h}$. Trials with distilled water as the lubricant are indicated with squares and plus signs, and trials with gel are marked with diamonds and crosses

sample including the reference value (distilled water) and the value of one of the obstetric gels.

At each condition, neither the friction forces of distilled water, the friction forces of obstetric gel, nor the pairwise differences followed a normal distribution (KolmogorovSmirnov test, alpha $=0.1)$. Consequently, a sign test was performed for each condition (different movement speeds, different dwell times) to determine whether the used obstetric gel significantly alters the friction force in comparison to distilled water.

For each sample, a relative reduction of friction force caused by using an obstetric gel was calculated as follows: The difference of friction force of gel minus the reference value (distilled water) was divided by the reference value.

The different movement velocities were evaluated with both gel A and gel B. A Wilcoxon rank sum test was performed for each movement speed to evaluate whether the two obstetric gels showed a significantly different relative reduction of the friction force. The whole analysis was done in MatLab and its statistical toolbox (Natick, Massachusetts, USA). All significance levels were set to $P<0.1$.

\section{Results}

\subsection{Modifications of movement velocities}

Overall, the measured friction forces ranged between 0.5 $7 \mathrm{~N}$. At a movement velocity of $10 \mathrm{~cm} / \mathrm{h}$, the use of gel A significantly reduced the friction force in comparison to distilled water. In contrast, lubrication with gel B resulted in higher dynamic friction forces in comparison to distilled water in four out of six samples, see Fig. 4. 
At a movement velocity of $50 \mathrm{~cm} / \mathrm{h}$, the reference trials with water always showed larger dynamic friction forces than trials with one of the obstetric gels, see Fig. 5. Both gels significantly reduced the dynamic friction force.

At the highest movement velocity of $100 \mathrm{~cm} / \mathrm{h}$, both obstetric gels significantly reduced the friction force when compared to distilled water, see Fig. 6.

At all movement velocities, gel A reduced the friction force significantly in relation to water by $30-40 \%$, see Table 2. At movement velocities of 50 and $100 \mathrm{~cm} / \mathrm{h}$, gel B showed relative reductions of the friction force in the same

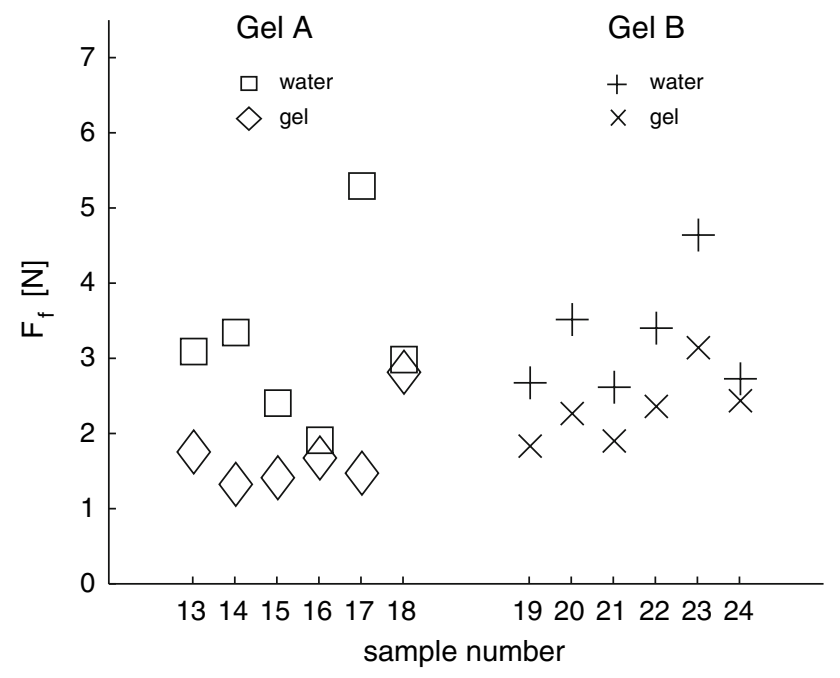

Fig. 5 Measured friction forces $F_{\mathrm{f}}$ for each sample moved at $50 \mathrm{~cm} / \mathrm{h}$ with no dwell time. Trials with distilled water as the lubricant are indicated with squares and plus signs, and trials with gel are marked with diamonds and crosses

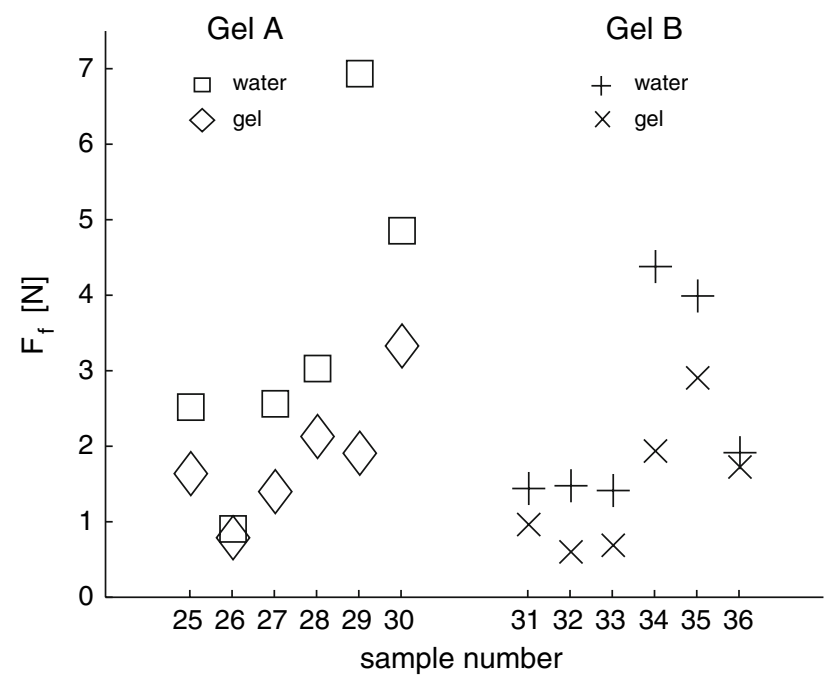

Fig. 6 Measured friction forces $F_{\mathrm{f}}$ for each sample moved at $100 \mathrm{~cm} /$ h. Trials with distilled water as the lubricant are indicated with squares and plus signs, and trials with gel are marked with diamonds and crosses magnitude (not significantly different, see Table 2, last column). However, at the slowest movement velocity of $10 \mathrm{~cm} / \mathrm{h}$, lubrication with gel $\mathrm{B}$ did not reduce the friction force in comparison to distilled water (see Table 2).

\subsection{Modification of dwell time}

The static friction forces measured during trials with distilled water ranged between 0.5 and $2.5 \mathrm{~N}$, whereas static friction forces of trials with gel A ranged only between 0.5 and $1 \mathrm{~N}$ (Fig. 7). With the exception of a dwell time of $30 \mathrm{~s}$, all other dwell time conditions resulted in significantly reduced static friction forces (Table 3).

\section{Discussion}

The presented work is the first one that quantifies the friction properties of obstetric gels being intended for facilitation of human childbirth. Human childbirth was simulated by applying similar mechanical conditions to a mammal tissue model. The presented procedure could not consider all influences of biomechanical conditions such as scalp hair moving against the vaginal wall or biochemical conditions such as hormones and $\mathrm{pH}$ value, which are usually altered during delivery. Nevertheless, the applied obstetric gels generally reduced the friction force in comparison to distilled water as a reference. The presented procedure thus confirmed the benefit of obstetric gels with respect to childbirth facilitation, which has already been observed in first clinical trials [12].

The reduction of the friction force by using an obstetric gel was significant in eight of ten test conditions. In the other two test conditions, the reduction of the friction force by using gels was not significant, which can be explained as follows:

Condition A: At a movement velocity of $10 \mathrm{~cm} / \mathrm{h}$, friction forces observed using gel $\mathrm{B}$ as a lubricant were in the same magnitude as the corresponding measurements with water (see Fig. 4). In contrast, gel A showed a significantly higher reduction of the friction force at this velocity (see Table 2). These results may be explained by the different viscosities of gel A (dynamic viscosity of $15 \mathrm{~Pa} \mathrm{~s}$ ) and gel $\mathrm{B}$ (5 Pa s): At a low movement velocity, separation of the counterparts seems to have a dominant impact on the friction force. Separation of the counterparts was reduced when the low-viscosity gel B was applied resulting in local stick effects, continuously re-occurring and distributed over the whole contact area. In sum, these local stick effects resulted in a friction force similar to that when distilled water was applied as a lubricant. Consequently, the low-viscosity gel could not reduce the friction force. At higher movement velocities, stick effects were 
Table 2 Median relative reduction and range of reduction of the friction force due to the use of an obstetric gel instead of water at the different movement speeds

\begin{tabular}{|c|c|c|c|c|c|}
\hline \multirow[t]{3}{*}{ Speed $(\mathrm{cm} / \mathrm{h})$} & \multicolumn{5}{|c|}{ Reduction of friction force due to obstetric gel in comparison to water (\%) } \\
\hline & \multicolumn{2}{|l|}{ Gel A } & \multicolumn{2}{|l|}{ Gel B } & \multirow[b]{2}{*}{$P$} \\
\hline & Median (\%) & Range $(\%)$ & Median (\%) & Range $(\%)$ & \\
\hline 10 & $29 *$ & $14-66$ & -5 & -19 to 9 & 0.002 \\
\hline 50 & $42 *$ & $6-72$ & $31 *$ & $11-36$ & 0.937 \\
\hline 100 & $33^{*}$ & $13-72$ & $42 *$ & $10-59$ & 0.310 \\
\hline
\end{tabular}

Six samples were measured for each condition. In the last column, the $P$ value of the Wilcoxon rank sum test is given comparing the magnitude of reduction of each gel

* Significant relative reduction $(P<0.1)$

Fig. 7 Summary of static friction forces $F_{\text {sf }}$ for different dwell times. Darker symbols represent longer dwell times; lubrication with water is marked with squares, and lubrication with gel A with diamonds

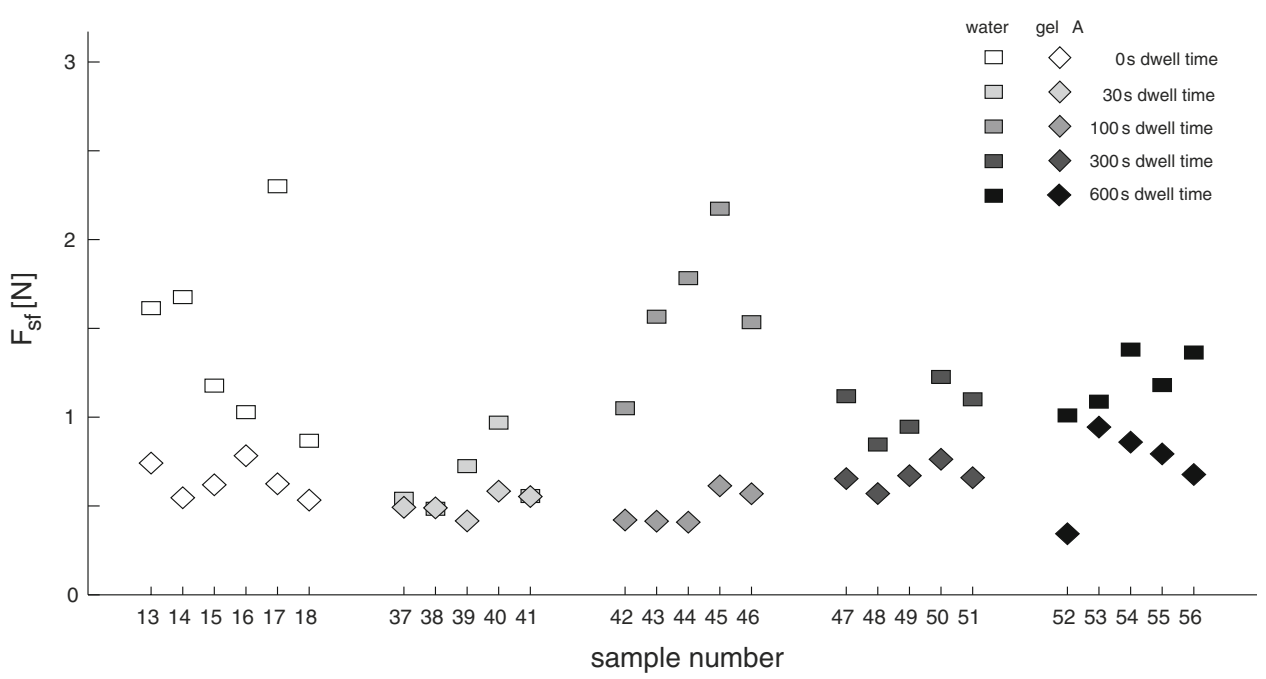

Table 3 Mean relative reduction of the friction force and its standard deviation SD due to the use of gel A instead of water at different dwell times

\begin{tabular}{llc}
\hline Dwell time (s) & \multicolumn{2}{l}{$\begin{array}{l}\text { Reduction of friction force due to gel A in } \\
\text { comparison to water }(\%)\end{array}$} \\
\cline { 2 - 3 } & Mean & SD \\
\hline 0 & $51^{*}$ & 18 \\
30 & 18 & 21 \\
100 & $69^{*}$ & 7 \\
300 & $36^{*}$ & 5 \\
600 & $40^{*}$ & 20
\end{tabular}

Five samples were measured for each condition

* Significant relative reduction $(P<0.1)$

not present; consequently, both obstetric gels reduced the friction forces in comparison to distilled water (applying water as a lubricant resulted in the worst separation of counterparts). These results are in agreement with other applications using lubricants. To reduce friction forces, i.e., within an engine, high-viscosity lubricants are also preferably used at low movement speeds (slow revolutions) [9].
Condition B: After a dwell time of $30 \mathrm{~s}$, gel A did not significantly reduce the static friction force in comparison to distilled water (Table 3). This non-significant reduction results from the lower static friction forces measured for distilled water after the dwell time of $30 \mathrm{~s}$ (force $<1 \mathrm{~N}$ ) than after all other dwell times (forces ranged from 1 to 2.5 N, Fig. 7). These lower static friction forces of distilled water were already in the magnitude of the commonly determined static friction forces measured during trials with gel A $(0.5-1.0 \mathrm{~N})$; as a consequence, no significant reduction was observed.

The presented procedure emphasizes that different quantities of ingredients can result in significantly different reductions of friction forces. However, before the presented procedure can be used to optimize the friction properties of obstetric gels according to the different stages of human childbirth, one major limitation of the procedure has to be solved: Currently, the experiments were done at room temperature, since at this stage of the project it was intended to make the benefits seen during clinical trials quantifiable. This lower than natural temperature may result in higher viscosities of the gels. As a consequence, at 
higher temperatures, both gels, and not only the low-viscosity gel B, may not reduce friction forces at low movement speeds. This has to be clarified in future experiments.

\section{Conclusion}

The presented procedure confirmed the benefit of obstetric gels with respect to childbirth facilitation that has been seen in clinical trials. The results further showed that differences in formulation of obstetric gels have an impact on the reduction of friction forces. This reduction was quantified by the presented procedure. Thus, in future investigations, the presented procedure can be used to optimize the friction properties of obstetric gels according to the different stages of human childbirth. The procedure cannot substitute clinical trials, but it can further help to minimize the number of required in vivo studies to find the optimal obstetric gel formulation. Furthermore, the presented procedure provides an objective point of view to ease the choice of obstetric gel for the different stages of labor.

Acknowledgments The authors wish to thank Giochen Bearth, abattoir's veterinary inspection service for providing fresh samples. The authors are further grateful to Mario Sikic and Diego Stutzer from the SMS-Lab and Rainer Burgkart from the University Hospital rechts der Isar, TU München, for their advice on this research.

\section{References}

1. Ahlers D (1993) Rechtsfragen in der Geburtshilfe. In: Richter/ Goetze Tiergeburtshilfe. Paul Parey, Berlin

2. Albers LL, Sedler KD, Bedrick EJ, Teaf D, Peralta P (2005) Midwifery care measures in second stage of labor and reduction of genital tract trauma at birth: a randomized trial. J Midwifery Womens Health 50:365-372. doi:10.1016/j.jmwh.2005.05.012
3. Brement S, Mossan S, Belery A, Racinet C (2008) Delivery in lateral position: randomized clinical trial comparing the maternal positions in lateral position and dorsal position for the second stage of labour. Gynecol Obstet Fertil 36:119-120. doi: 10.1016/j.gyobfe.2007.10.021

4. Cheng YW, Hopkins LM, Caughey AB (2004) How long is too long: does a prolonged second stage of labor in nulliparous women affect maternal and neonatal outcomes? Am J Obstet Gynecol 2004191:933-938. doi:10.1016/j.ajog.2004.05.044

5. Cheng YW, Hopkins LM, Russel KL, Aaron BC (2007) Duration of the second stage of labor in multiparous women: maternal and neonatal outcomes. Am J Obstet Gynecol 196:585-587

6. Donnelly V, Fynes M, Campbell D, Johnson H, O'Connell PR, O'Herlihy C (1998) Obstetric events leading to anal sphincter damage. Obstet Gynecol 92:955-961. doi:10.1016/S0029-7844 (98)00255-5

7. Eason E, Labrecque M, Wells G, Feldman P (2000) Preventing perineal trauma during childbirth: a systematic review. Obstet Gynecol 95:464-471. doi:10.1016/S0029-7844(99)00560-8

8. Hofmeyr GJ (2004) Obstructed labor: using better technologies to reduce mortality. Int J Gynaecol Obstet 85(S1):S62-S72. doi: 10.1016/j.ijgo.2004.01.011

9. Klingelnerg J (2008) Kegelräder: Grundlagen, Anwendungen. Springer, Berlin

10. Leypold K (2008) Experimental investigations on friction and lubrication during delivery. Master thesis, Departement of Medical Engineering, TU München

11. Rempen A, Kraus M (1991) Pressures on the fetal head during normal labor. J Perinat Med 19:199-206

12. Schaub AF, Litschgi M, Hoesli I, Holzgreve W, Bleul U, Geissbühler V (2008) Obstetric gel shortens second stage of labor and prevents perineal trauma in nulliparous women: a randomized controlled trial on labor facilitation. J Perinat Med 36:129135. doi:10.1515/JPM.2008.024

13. Stamp G, Kruzins G, Crowther C (2001) Perineal massage in labour and prevention of perineal trauma: randomised controlled trial. BMJ 322:1277-1280. doi:10.1136/bmj.322.7297.1277

14. Stewart P, Hillan E, Calder AA (1983) A randomized trial to evaluate the use of a birth chair for delivery. Lancet 1:12961299. doi:10.1016/S0140-6736(83)92412-1

15. Turner MJ, Romney NL, Webb JB, Gordon H (1986) The birthing chair: an obstetric hazard? J Obstet Gynaecol 6:232-235. doi:10.3109/01443618609079206

16. Waldenstrom U, Gottvall K (1991) A randomized trial of birthing stool of conventional semirecumbent position for second stage of labor. Birth 18:5-10. doi:10.1111/j.1523-536X.1991.tb00045.x 